\title{
Heart Murmur Detection using Fractal Analysis of Phonocardiograph Signals
}

\author{
Anandarup Mukherjee \\ University of Engineering \& \\ Management \\ Jaipur \\ India
}

\author{
Nidhi Pathak \\ University of Engineering \& \\ Management \\ Jaipur \\ India
}

\author{
Anirban Roy \\ University of Engineering \& \\ Management \\ Jaipur \\ India
}

\begin{abstract}
This paper proposes a method of differentiating normal heart sounds S1 and S2 from those of a heart with valvular dysfunction by comparing the generated Fractal dimension using Higuchi's Fractal Dimension. The aim is to provide a classifying parameter which can clearly demarcate normal heart sounds from those with murmurs. The performance of this method is verified against a Continuous Wavelet transform generated time- scale domain method.
\end{abstract}

\section{General Terms}

Fractal Dimension, Phonocardiograph, Neural Networks.

\section{Keywords}

Fractal Dimension, Higuchi's Algorithm, Phonocardiograph, Heart Sounds, Murmurs

\section{INTRODUCTION}

Diagnosis of the heart sounds and murmurs have been employing the use of different methods like spectral tracking, heart sound energy, wavelet based method and other such approaches for a long time. There have been many proposed methods to interpret, differentiate and separate the constituting elements of a phonocardiograph cycle to help us recognize various cardiac dysfunctions that substantially affect the sounds produced by the human heart. None of these

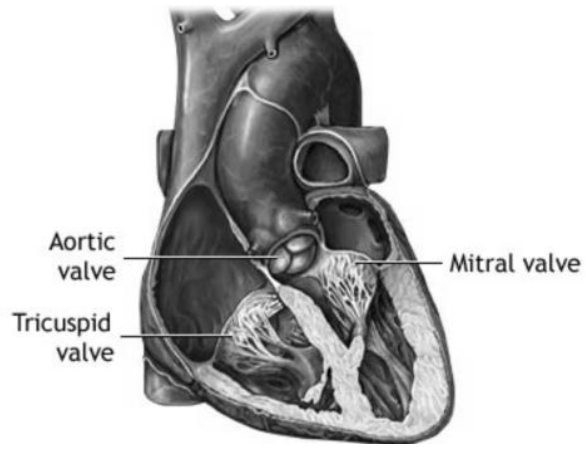

Fig 1: A cross section of the human heart depicting the positions of various valves.

techniques establishes any strong or vital methodology to analyze the heart sounds efficiently. Spargue used different types of electronic high frequency stethoscopes to record the human heart sounds [1]. The present day world has many electronic devices, algorithms and processes to detect and record the heart sounds. Methods like wavelet based identification of heart sounds using spectrogram analysis [2] are common. Regardless of the developed technology and available techniques involving digital signal processing, the erratic and complex nature of heart sounds make it difficult to accurately detect some of the diseased and insubstantial sounds which may go unnoticed.

Heart auscultation is the process of studying heart sounds and mumbles. Cardiac auscultation helps in the study of cardiac cycles and its related dysfunctions or diseases, if any. These sounds are produced due to the activity of the cardiac valves and the vessels like Pulmonary arteries, Pulmonary veins, and Aorta. The major cardiac valves are shown in figure- 1 . The heart sounds are obtained using microphones that are placed on the thorax, in the main auscultatory areas. There are two types of sounds, the high frequency sounds caused by the opening and closing of the valves and the low frequency sounds associated with other ventricular activities. The low frequency sounds generally correspond to an abnormality and can be further divided into extra beats and murmurs [2]. The classification of human cardiac auscultatory sounds is given in sub-sections 1.1 to 1.5 .

\subsection{First Heart Sound (S1)}

The first heart sound or S1 comprises of the "lub" sound of the normal "lub-dub" heart sound pair as shown in figure-2. The beginning of the systole starts with ventricular contraction causing the papillary muscles connected to them to stretch. These papillary muscles are attached to the Mitral and Tricuspid valves by the Chordae Tendineae. The contraction of ventricles result in the closure of these valves

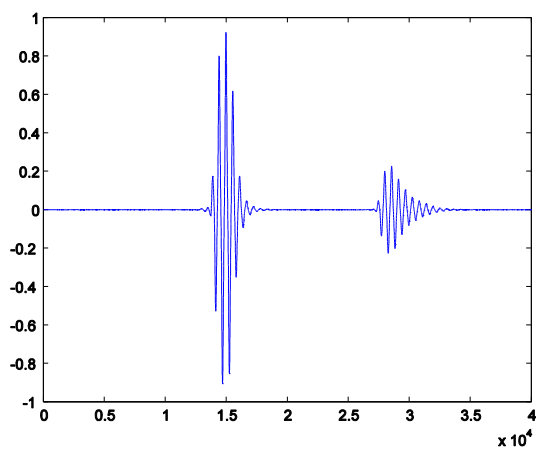

Fig 2: A PCG recording of normal S1-S2 complex

[3]. The contraction of ventricles results in the rise of intraventricular pressure which surpasses the atrial pressure. The Chordae Tendineae prevents the valves from driving into the atria due to pressure rise in the ventricles. The sudden closure of the AV (Atrio Ventricular) valves and the contraction of 
ventricles restrict the regurgitation i.e., the reverse flow of the blood back into the Atria. This abrupt blockage of reverse flow of blood reverberates in the ventricles which produces the first heart sound $\mathrm{S} 1$. The sound $\mathrm{S} 1$ is further divided into two components, M1 and T1 which correspond to the closure of Mitral and Tricuspid valves respectively. These components act as indicators to the functioning of the heart. For example, the precedence of T1 over M1 is a strong indicator of left sided cardiac diseases such as Left Bundle Branch block (LBBB).

\subsection{Second Heart Sound (S2)}

The second heart sound S2 comprises the "dub" sound of the "lub-dub" pair. This sound occurs after the iso-volumetric relaxation period at the end of systole and the beginning of diastole. This sound is further divided into components A2 and P2. The component A2 is due to the closure of Aortic valves and $\mathrm{P} 2$ due to the closure of Pulmonary valves [3]. The sound S2 is generated due to the blockage of the reverse flowing blood caused by the closure of the semi-lunar valves at the beginning of the relaxation of the ventricles i.e., ventricular diastole. The draining of blood from the left ventricle causes the ventricular pressure to fall below the Aortic pressure. This pressure difference tries to drive the blood back towards the left ventricle but is stopped by the closed Aortic valves. Similarly, in case of the right ventricle, the pressure of the Pulmonary artery exceeds the pressure of the right ventricle resulting in reverse blood flow, which is subsequently obstructed by the closure of pulmonary valves causing reverberation within the heart, causing S2.

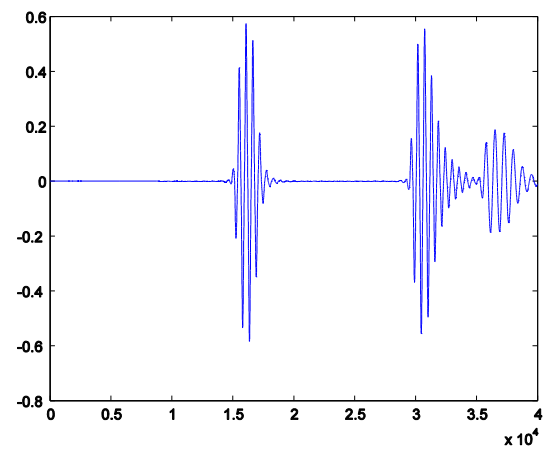

Fig 3: A PCG recording, depicting S1, $\mathrm{S} 2$ and $\mathrm{S3}$ sounds (in sequential order)

The sound S2 is depicted in figure-2. The splitting of S2 sound is often heard during the process of inspiration but a widely split $\mathrm{S} 2$ can be a sign of cardio vascular dysfunction such as Right Bundle Branch block (RBBB), Pulmonary Stenosis or Atrial Septal defect.

\subsection{Third Heart Sound (S3)}

The third heart sound is a rare extra sound, heard just after S2 as shown in figure-3. It occurs at the beginning of diastole which gives it the name proto-diastolic or ventricular gallop [4]. This sound may appear as normal in people below 40 years of age and athletes but when detected in old age, may be a symptom of some serious problem such as heart failure. The primary cause of this sound is rapid, increased or over filling of the ventricles. During this rapid filling of ventricles, the blood from atria reverberates back and forth within the walls of the ventricles [5]. There are many conditions that may lead to the generation of S3. This sound may occur due to Mitral regurgitation in which the Mitral valves fail to stop the blood flow into the left atrium from the left ventricle leading to overfilled atrium which in turn results in rapid ventricular filling upon opening of the Mitral valve. Stiffened and dilated ventricular walls also result in increased filling pressure. Other conditions which may result in the third heart sound may include the ventricular septal defect (the presence of a hole between the ventricular walls allowing for the rapid filling from one ventricle to another), dilated cardio-myopathy (reduced flexibility of the walls of the left ventricles), death of the ventricular tissue due to insufficient blood supply causing Hypokinesia and Akinesia, Tricuspid regurgitation, etc.

\subsection{Fourth Heart Sound (S4)}

The fourth heart sound is another extra sound which occurs just before S1 due to a condition called Hypertrophic ventricle where, the atria contracts briskly, attempting to surmount an abnormally stiff ventricle. It is also referred to as Atrial/ presystolic gallop as it occurs in the late cardiac cycle. The position of S4 with respect to sounds $\mathrm{S} 1$ and $\mathrm{S} 2$ is depicted in figure-4. It is estimated that this sound is produced when blood from the atria is forced into a ventricle with stiffened walls, resulting in an abnormal and turbulent flow. A loud S4 is generally associated with the pathological condition of left ventricular failure. The cause of this sound includes any condition that increases the stiffness of the ventricular walls such as hypertrophy of the ventricles, chronic hypertension leading to hypertrophic ventricles, ventricular overloading or fibrosis. In case of Tachycardia, it becomes difficult to differentiate between $\mathrm{S} 3$ and $\mathrm{S} 4$.

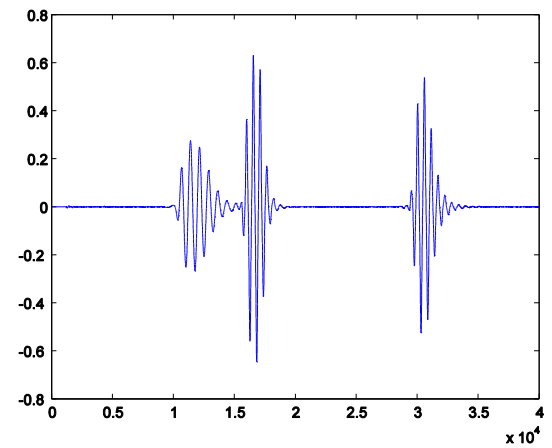

Fig 4: A PCG recording, depicting the heart sounds S4, S1 and $S 2$ (in sequential order)

\subsection{Heart Murmurs}

Cardiac murmurs are produced due to the turbulent flow of blood through the heart which is tumultuous enough to create an audible gurgling noise. This sound is almost inaudible for most of the cardiac dysfunctions and diseases. Murmurs, most commonly heard in adults are due to blood flowing in the opposite direction through the Mitral valve of the heart (regurgitation) [2]. Even though the volume of the blood flow is small but the sound is quite audible. Narrowing and stiffening of the Aortic valve commonly known as Stenosis, causes a systolic ejection which is one of the most common forms of murmur [6]. 


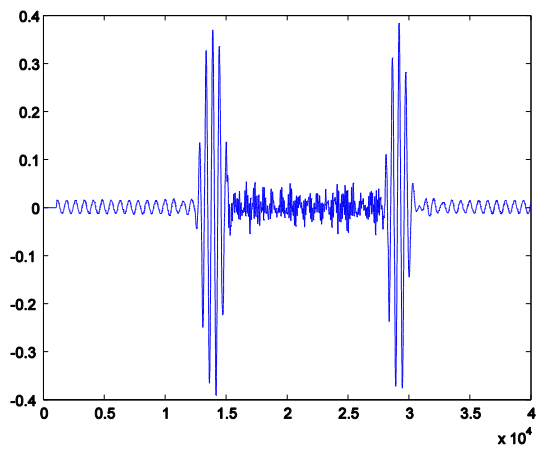

Fig 5: A PCG recording of an S1-S2 complex with holosystolic murmur. The disturbance before, between and after the complex is caused due to the murmur

This sound is common in aged persons and people with two Leaflet valves instead of three. Mitral valve stenosis sometimes produces soft murmurs audible with high quality electronic stethoscopes. Other audible murmurs are due to the abnormal opening and closing of valves between the left and right ventricles. A sample case of cardiac murmurs caught on PCG is depicted in figure-5.

\section{FRACTAL ANALYSIS}

Fractal dimension gives the basic building block of any pattern or object; it gives the complexity and the measure of self-similarity in an object. Since, no physical object is completely self-similar, i.e., absence of self-similarity at all scales; fractal dimension gives the extent to which the object follows self-similarity, which may be over spatial or temporal scales. There are many theories or ideas regarding the fractal characteristics of an object such as, the Hausdorff dimension, Renyi dimension, Packing dimension, etc., famous for their theoretical importance. Theories like Correlation dimension and Box counting dimension [7] are extensively used in practice due to their ease of application. According to fractal geometry, the fractal dimension gives the space filling capacity for any object. The fractal dimension of an object may or may not be an integer [8].

\subsection{Higuchi Fractal Dimension}

Fractal dimension (FD) is a notion or theory of describing the structure and complexity of natural objects. It illustrates the deformity of a time series. Fractal Dimension describes an object or pattern as a collection of geometric figures arranged in a fashion that forms the object. This dimension is the detailed or the most intricate part of the massive figure under observation. The term 'Fractal' comes from a Latin word 'fractus' which means broken or uneven [8]. Thus, FD is that smallest broken part or unit of an object which when pulled together can spawn the object. There are many methods to find the fractal dimension of an object. These include the Katz, Sevcik and Higuchi methods. We have considered the Higuchi's method of finding the fractal dimension. In this method, a time series data is taken to get different sets of observations at regular intervals-

$$
X(1) X(2) X(3) \ldots \ldots X(N)
$$

For this given time series a new time series is constructed-

$$
X_{k}^{m}=X(m) X(m+k) X(m+2 k) \ldots . . X\left(m+\left[\frac{N-m}{k}\right] k\right)
$$

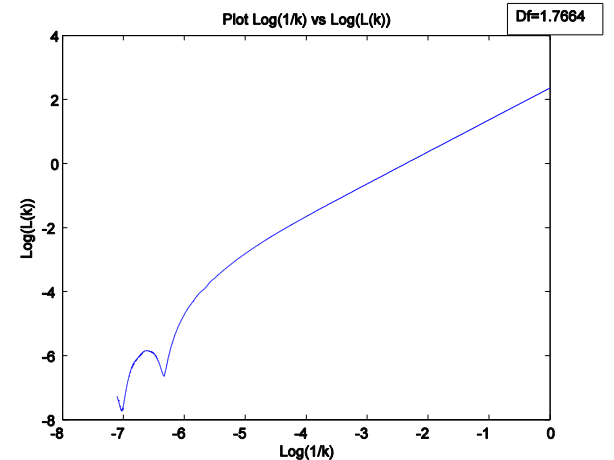

Fig 6: A log-log plot of length of curve vs. the inverse of time interval showing the result of application of Higuchi's algorithm on a PCG recording of normal S1-S2 complex to determine the fractal dimension of the complex

Here $m$ is the initial time $(m=1,2,3 \ldots)$ and $k$ is the time interval. For $k$ different intervals we get $k$ different time series from the above process. For each of these series the length of the corresponding curve is calculated using the following formula:-

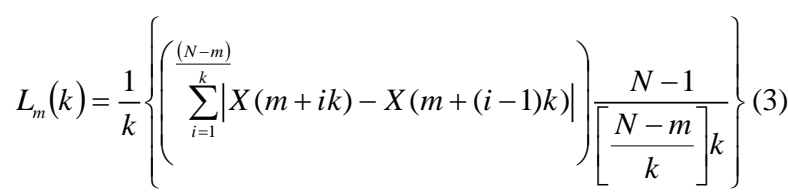

The curve length is normalized and the average curve length over all the $k$ sets of time series length $L_{m}(k)$ is taken as $\langle L(k)\rangle$. The curve is said to be a fractal curve if $\langle L(k)\rangle \propto k^{-D}$ where $\mathrm{D}$ is the required fractal dimension [9].

\section{DATABASE}

The data for the PCG analysis is taken from the University of Michigan, Heart Sound \& Murmur library [10]. The database is divided into four main sections based on the method or region of sound acquisition. These sections can be listed as:

\subsection{Apex Area I}

The patient is supine for the duration of data acquisition. The data is recorded using the Bell of the stethoscope.

\subsection{Apex Area II}

The patient is in the left decubitus position for the duration of data acquisition. The data is recorded using the Bell of the stethoscope.

\subsection{Aortic Area}

The patient is sitting and the data is recorded using the Bell of the stethoscope.

\subsection{Pulmonic Area}

The patient is supine and the data is recorded using the diaphragm of the stethoscope.

Each of these sections consists of various samples of PCG signals from a wide variety of patients. The files are encoded in mp3 format with a bit rate of $128 \mathrm{kbps}$. Each of these recordings is approximately 65 to 70 seconds long.

\section{METHODOLOGY}

Four types of records are taken from the database viz., the normal S1 and S2 record, early systolic murmur, mid systolic murmur and holo- systolic murmur, recorded at the apex 
region of the chest. The sounds are first manually segregated into complexes comprising of s1 and s2 to capture the systolic information of the heart.

The first method comprises of expanding the signal complexes in the frequency domain using Continuous Wavelet Transform (CWT) [11][12], choosing the principal components to reduce the dimensionality of the signal using Principal Component Analysis (PCA) and then classifying it using a Neural Network classifier with 10 hidden layers and employing back-propagation algorithm.

Gnitecki applied Katz, modified Katz and Variance FD algorithms in the study of lung sound fractality [13]. Our second approach involves subjecting the signal complexes to the HFD algorithm in order to determine the fractal dimension of the complexes. The fractal dimensions of the complexes are treated as inputs to a Neural Network classifier. The plot of curve length versus the inverse of time interval upon application of HFD on a PCG S1-S2 complex to determine its dimension is shown in figure-6.

\section{RESULTS}

The result of application of HFD on the above mentioned complexes are tabulated in table- 1 for all the chosen heart sounds.

Table 1: The comparative HFD values of Normal heart sound, early systolic murmur, mid systolic murmur and holo systolic murmur for all 52 detected S1- S2 complexes.

\begin{tabular}{|c|c|c|c|c|}
\hline \multirow[b]{2}{*}{ Complex } & \multicolumn{4}{|c|}{ HFD Values } \\
\hline & Normal & $\begin{array}{c}\text { Early } \\
\text { Systolic } \\
\text { Murmur }\end{array}$ & $\begin{array}{c}\text { Mid } \\
\text { Systolic } \\
\text { Murmur }\end{array}$ & $\begin{array}{c}\text { Holo } \\
\text { Systolic } \\
\text { Murmur }\end{array}$ \\
\hline Complex1 & 1.9396 & 1.9526 & 1.969 & 1.9637 \\
\hline Complex2 & 1.9276 & 1.9403 & 1.9683 & 1.9564 \\
\hline Complex3 & 1.9384 & 1.9538 & 1.9688 & 1.9657 \\
\hline Complex 4 & 1.9226 & 1.9481 & 1.9632 & 1.9564 \\
\hline Complex 5 & 1.9353 & 1.9543 & 1.9554 & 1.9675 \\
\hline Complex6 & 1.9411 & 1.9501 & 1.9703 & 1.9637 \\
\hline Complex7 & 1.9381 & 1.9537 & 1.9678 & 1.9564 \\
\hline Complex8 & 1.9401 & 1.9515 & 1.966 & 1.9657 \\
\hline Complex 9 & 1.9271 & 1.9531 & 1.9659 & 1.9563 \\
\hline $\begin{array}{c}\text { Complex } 1 \\
0\end{array}$ & 1.9384 & 1.9526 & 1.9686 & 1.9674 \\
\hline $\begin{array}{c}\text { Complex } 1 \\
1\end{array}$ & 1.9413 & 1.9453 & 1.9692 & 1.9637 \\
\hline Complex 1 & 1.9364 & 1.9538 & 1.9702 & 1.9564 \\
\hline $\begin{array}{c}\text { Complex } 1 \\
3\end{array}$ & 1.9408 & 1.948 & 1.967 & 1.9657 \\
\hline$\underset{4}{\text { Complex } 1}$ & 1.9321 & 1.9543 & 1.9648 & 1.9562 \\
\hline Complex 1 & 1.9399 & 1.95 & 1.965 & 1.9673 \\
\hline
\end{tabular}

\begin{tabular}{|c|c|c|c|c|}
\hline $\begin{array}{c}\text { Complex } 1 \\
6\end{array}$ & 1.9289 & 1.9538 & 1.9688 & 1.9637 \\
\hline $\begin{array}{c}\text { Complex1 } \\
7\end{array}$ & 1.9379 & 1.9514 & 1.9693 & 1.9563 \\
\hline $\begin{array}{c}\text { Complex1 } \\
8\end{array}$ & 1.9412 & 1.9531 & 1.9639 & 1.9657 \\
\hline $\begin{array}{c}\text { Complex1 } \\
9\end{array}$ & 1.9361 & 1.9525 & 1.9693 & 1.9562 \\
\hline $\begin{array}{c}\text { Complex } 2 \\
0\end{array}$ & 1.9412 & 1.9527 & 1.9691 & 1.9673 \\
\hline $\begin{array}{c}\text { Complex2 } \\
1\end{array}$ & 1.9333 & 1.9537 & 1.9699 & 1.9637 \\
\hline $\begin{array}{c}\text { Complex } 2 \\
2\end{array}$ & 1.9398 & 1.9479 & 1.9661 & 1.8634 \\
\hline $\begin{array}{c}\text { Complex2 } \\
3 \\
\end{array}$ & 1.9289 & 1.9543 & 1.9636 & 1.9657 \\
\hline $\begin{array}{c}\text { Complex2 } \\
4\end{array}$ & 1.938 & 1.9499 & 1.97 & 1.9561 \\
\hline $\begin{array}{c}\text { Complex } 2 \\
5\end{array}$ & 1.9421 & 1.9538 & 1.9688 & 1.9672 \\
\hline $\begin{array}{c}\text { Complex2 } \\
6\end{array}$ & 1.9364 & 1.9514 & 1.968 & 1.9637 \\
\hline $\begin{array}{c}\text { Complex2 } \\
7 \\
\end{array}$ & 1.941 & 1.9531 & 1.9684 & 1.9562 \\
\hline $\begin{array}{c}\text { Complex2 } \\
8\end{array}$ & 1.9323 & 1.9525 & 1.9682 & 1.9657 \\
\hline $\begin{array}{c}\text { Complex2 } \\
9\end{array}$ & 1.9392 & 1.9527 & 1.9685 & 1.956 \\
\hline $\begin{array}{c}\text { Complex3 } \\
0\end{array}$ & 1.9417 & 1.9537 & 1.9701 & 1.9671 \\
\hline $\begin{array}{c}\text { Complex3 } \\
1\end{array}$ & 1.9368 & 1.9478 & 1.9685 & 1.9636 \\
\hline $\begin{array}{c}\text { Complex3 } \\
2 \\
\end{array}$ & 1.9418 & 1.9543 & 1.9673 & 1.9561 \\
\hline $\begin{array}{c}\text { Complex3 } \\
3 \\
\end{array}$ & 1.9343 & 1.9498 & 1.9675 & 1.9656 \\
\hline $\begin{array}{c}\text { Complex3 } \\
4\end{array}$ & 1.9404 & 1.9538 & 1.9685 & 1.956 \\
\hline $\begin{array}{c}\text { Complex3 } \\
5 \\
\end{array}$ & 1.9299 & 1.9513 & 1.9687 & 1.9669 \\
\hline $\begin{array}{c}\text { Complex3 } \\
6\end{array}$ & 1.9387 & 1.9531 & 1.9702 & 1.9636 \\
\hline $\begin{array}{c}\text { Complex3 } \\
7 \\
\end{array}$ & 1.9417 & 1.9524 & 1.968 & 1.9561 \\
\hline $\begin{array}{c}\text { Complex3 } \\
8\end{array}$ & 1.935 & 1.9527 & 1.9664 & 1.9656 \\
\hline $\begin{array}{c}\text { Complex3 } \\
9\end{array}$ & 1.941 & 1.9536 & 1.9664 & 1.956 \\
\hline $\begin{array}{c}\text { Complex } 4 \\
0\end{array}$ & 1.9316 & 1.9477 & 1.9685 & 1.9668 \\
\hline $\begin{array}{c}\text { Complex } 4 \\
1 \\
\end{array}$ & 1.9393 & 1.9543 & 1.9691 & 1.9636 \\
\hline $\begin{array}{c}\text { Complex4 } \\
2\end{array}$ & 1.926 & 1.9498 & 1.9696 & 1.956 \\
\hline $\begin{array}{c}\text { Complex4 } \\
3 \\
\end{array}$ & 1.9383 & 1.9539 & 1.9653 & 1.9429 \\
\hline $\begin{array}{c}\text { Complex4 } \\
4\end{array}$ & 1.9414 & 1.9513 & 1.9679 & 1.9644 \\
\hline $\begin{array}{c}\text { Complex4 } \\
5\end{array}$ & 1.9332 & 1.9531 & 1.9632 & 1.9667 \\
\hline $\begin{array}{c}\text { Complex4 } \\
6\end{array}$ & 1.9396 & 1.9524 & 1.9693 & 1.9635 \\
\hline $\begin{array}{c}\text { Complex } 4 \\
7 \\
\end{array}$ & 1.9415 & 1.9527 & 1.969 & 1.9642 \\
\hline
\end{tabular}




\begin{tabular}{|c|c|c|c|c|}
\hline $\begin{array}{c}\text { Complex4 } \\
8\end{array}$ & 1.9376 & 1.9536 & 1.9692 & 1.9655 \\
\hline $\begin{array}{c}\text { Complex4 } \\
9\end{array}$ & 1.941 & 1.9519 & 1.9641 & 1.9645 \\
\hline $\begin{array}{c}\text { Complex5 } \\
0\end{array}$ & 1.9336 & 1.9543 & 1.9659 & 1.9666 \\
\hline $\begin{array}{c}\text { Complex5 } \\
1\end{array}$ & 1.9396 & 1.9497 & 1.9694 & 1.9634 \\
\hline $\begin{array}{c}\text { Complex5 } \\
2\end{array}$ & 1.9414 & 1.9539 & 1.9692 & 1.9644 \\
\hline
\end{tabular}

record to get a simple and clear understanding of the distribution of the HFD values corresponding to each condition, as shown in Table- 2 . The minimum and maximum values of the four record sets may overlap, forming no clear or distinctive boundary. The inclusion of mean and standard deviation in table-2 makes it easier to predict the subsequent HFD value and hence, the S1-S2 complex type most likely to occur after a certain complex has already occurred. This is used for identifying the dataset to which a complex may belong to.

Table-1 can be summarized with the help of minimum maximum, standard deviation and mean values for each

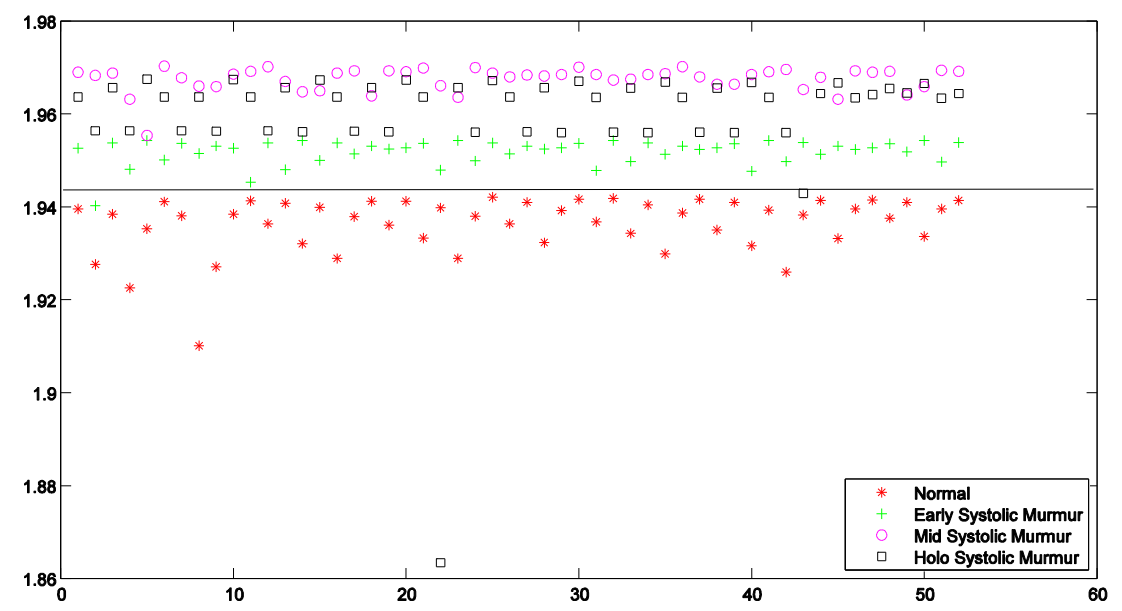

Fig 7: A comparative plot of all the generated data points after application of HFD to the all four classes of signals

Figure 7 shows the comparative plot of the distribution of the HFD values of the four signals. The signals with murmurs form a clear boundary as emphasized by the solid demarcation line. This boundary roughly segregates the normal heart sounds from the sounds with murmurs. It is also seen in figure-7 that records with early systolic murmur and mid systolic murmur also form a distinctive boundary from each other.

\section{CONCLUSION}

The classification of normal and dysfunctional auscultatory heart sounds employing time- scale based expansion and dimensionality reduction using PCA gives abysmally poor performance. The classification of Normal complexes from complexes with holo- systolic murmur gives an accuracy of around $57.2 \%$. This accuracy signifies that the classifier is

Table 2: Summarization of Table-1 with respect to minimum, maximum, mean value and standard deviation for each of the four classes of heart sounds taken.

\begin{tabular}{|c|c|c|c|c|}
\hline & Minimum & Maximum & Mean & $\begin{array}{c}\text { Standard } \\
\text { Deviation }\end{array}$ \\
\hline Normal & 1.9101 & 1.9421 & 1.9364 & 0.0061 \\
\hline $\begin{array}{c}\text { Early } \\
\text { Systolic } \\
\text { Murmur }\end{array}$ & 1.9403 & 1.9543 & 1.9518 & 0.0027 \\
\hline $\begin{array}{c}\text { Mid } \\
\text { Systolic }\end{array}$ & 1.9554 & 1.9703 & 1.9676 & 0.0026 \\
\hline
\end{tabular}

\begin{tabular}{|c|c|c|c|c|}
\hline Murmur & & & & \\
\hline $\begin{array}{c}\text { Holo } \\
\text { Systolic } \\
\text { Murmur }\end{array}$ & 1.8634 & 1.9675 & 1.9600 & 0.0146 \\
\hline
\end{tabular}

bound to misclassify normal heart sounds as those with murmurs or vice versa, approximately half of the time. The result for this method is shown in figure- 8 with the help of a confusion matrix and a Receiver Operating Characteristics (ROC) curve. 

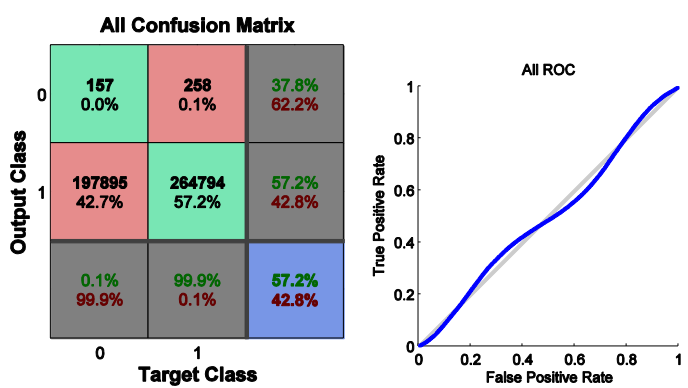

Fig 8: The confusion matrix and the Receiver Operating Characteristics of the classifier after application of the CWT based method
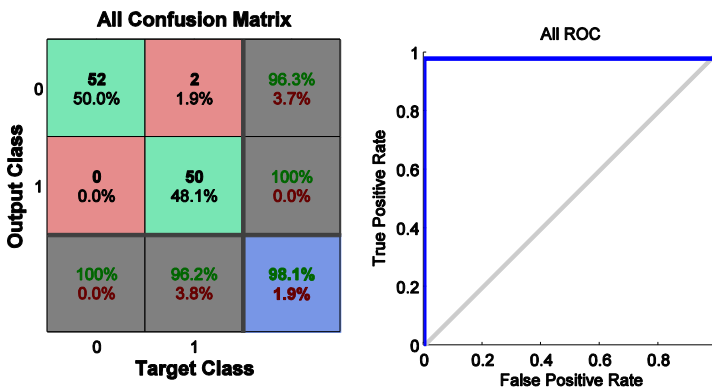

Fig 9: The confusion matrix and the Receiver Operating Characteristics of the classifier after application of HFD based method

The HFD values, when used for classifying the dysfunctional heart sounds from the normal ones, using a neural network classifier, gives a very good accuracy of classification. The classification of normal heart sound from early systolic murmur, mid systolic murmur and holo- systolic murmur gives an accuracy of $100 \%, 99 \%$ and $98.1 \%$ respectively. This result is as expected since it is clear from the plot in figure 7 that the data points of auscultatory normal heart sound form a clear boundary away from the data points of sounds with murmurs. Figure- 9 shows the confusion matrix and the ROC curve for classification between normal heart sounds and sounds with holo-systolic murmur.

\section{REFERENCES}

[1] Howard B. Sprague, M.D., Patrick A. Ongley, M.D., "The Clinical Value of Phonocardiography", Circulation. 1954;9:127-134

[2] Nilanjan Dey, Achintya Das, Sheli Sinha Chaudhuri, "Wavelet Based Normal and Abnormal Heart Sound Identification using Spectrogram Analysis," International Journal of Computer Science \& Engineering Technology (IJCSET), Vol. 3, No. 6, June 2012

[3] Todd R. Reed, Nancy E. Reed, Peter Fritzson," Heart sound analysis for symptom detection and computeraided diagnosis," Simulation Modeling Practice and Theory 12 (2004) 129-146

[4] Mark E. Silverman, "The Third Heart Sound", Chapter 24, Clinical Methods: The History, Physical, and Laboratory Examinations. $3^{\text {rd }}$ Edition, Boston: Butterworths; 1990
[5] Patel R, Bushnell DL, Sobotka PA: Implications of an audible third heart sound in evaluating cardiac function, The Western Journal Of Medicine, June 1993

[6] Heart murmur and sounds, Medline Plus, http://www.nlm.nih.gov/medlineplus/ency/article/003266 .htm

[7] B. S. Raghavendra, and D. Narayana Dutt, "Computing Fractal Dimension of Signals using Multiresolution Boxcounting Method", World Academy of Science, Engineering and Technology 372010.

[8] Benoit B. Mandelbrot, "The fractal geometry of nature", Henry Holt and company, 1983

[9] T. Higuchi, "Approach to an irregular time series on the basis of the fractal theory", Physica D 31(1988).

[10] University of Michigan, Heart Sound and Murmur Library, http://www.med.umich.edu/lrc/psb/heartsounds/.

[11] A. Mukherjee, K. K. Ghosh, "A Noise Independent Approach to QRS-Complex Detection Using CWT", International Conference on Informatics, Electronics and Vision, Dhaka, Bangladesh. 18-19 May 2013, IEEE Xplore (Digital Object Identifier: 10.1109/ICIEV.2013.6572722), p.1-5, 2013.

[12] Abdallah M. El-Ramsisi, Hassan A. Khalil, "Diagnosis System Based on Wavelet Transform, Fractal Dimension and Neural Network", Journal of Applied Sciences 7 (24):3971-3976, 2007, ISSN 1812-5654.

[13] J. Gnitecki, Z. Moussami, "The fractality of lung sounds: A comparison of three waveform fractal dimension algorithms", Chaos, Solitons \& Fractals, Elsevier 2005, DOI: 10-1016/j.chaos.2005.02.018, 0960-0779.

\section{AUTHOR'S PROFILE}

Anandarup Mukherjee (Member, IEEE) completed his Master's with specialization in Microelectronics \& VLSI Design in 2012. He is currently an Assistant Professor in the department of Electronics \& Communication Engineering at the University of Engineering \& Management, Jaipur, India. His research interests include Bio-Medical Signal Processing, Robotics, Data Mining, Pattern Analysis and Steganography. $\mathrm{He}$ regularly writes for various scientific journals and magazines and has 13 journal and conference publications till date.

Nidhi Pathak is a third year student, pursuing her Bachelor's degree from University of Engineering \& Management, Jaipur, India, in the department of Electronics \& Communication Engineering. Her area of interests includes Bio-Medical Signal Processing and statistical analysis. She regularly contributes research papers and articles to various conferences and journals.

Anirban Roy is a second year student, pursuing his Bachelor's degree in the field of Electronics and Communication Engineering from the University of Engineering and Management, Jaipur, Rajasthan, India. His areas of interests include Embedded Systems, Robotics, Digital Signal Processing and Bio-Medical Signal Processing. He regularly works on hardware as well as software based applications of Signal Processing and aspires to become a researcher in the same. 